\title{
Germanica
}

\section{Max Beckmann ou la réinvention de la beauté}

Von der Schönheit zur Hässlicheit, Max Beckmanns Tagebücher

\section{Florence Bancaud}

\section{(2) OpenEdition Journals}

Édition électronique

URL : http://journals.openedition.org/germanica/438

DOI : 10.4000/germanica.438

ISSN : 2107-0784

Éditeur

Université de Lille

\section{Édition imprimée}

Date de publication : 1 décembre 2005

Pagination : 147-165

ISBN : 2-913857-16-7

ISSN : 0984-2632

\section{Référence électronique}

Florence Bancaud, « Max Beckmann ou la réinvention de la beauté », Germanica [En ligne], 37 | 2005, mis en ligne le 07 janvier 2010, consulté le 10 décembre 2020. URL : http://journals.openedition.org/ germanica/438; DOI : https://doi.org/10.4000/germanica.438

Ce document a été généré automatiquement le 10 décembre 2020.

(c) Tous droits réservés 


\title{
Max Beckmann ou la réinvention de la beauté
}

Von der Schönheit zur Hässlicheit, Max Beckmanns Tagebücher

\author{
Florence Bancaud
}

1 «Inventer la beauté en distillant l'horreur ${ }^{1}$ ». Ces propos d'Alfred Kubin pourraient parfaitement s'appliquer à Max Beckmann, ce peintre inclassable et contradictoire, férocement jaloux de son individualité et pourtant épigone à ses débuts, ce témoin critique de son temps qui prône pourtant le dépassement métaphysique du réel. Son oscillation constante entre beauté et laideur, espoir et pessimisme, hédonisme et mélancolie, s'explique par un rapport des plus ambigus avec la réalité. Pour Philippe Dagen, il incarne l'artiste qui est « à la fois dans et hors le monde; celui qui, dans une position intenable, ne peut s'empêcher d'aimer cette réalité qui le menace de disparition et ne peut résoudre cette contradiction. Deux mouvements opposés l'animent : l'attraction et l'horreur ${ }^{2}{ }^{\prime}$.

Dans la polémique qui l'oppose en 1912 à Franz Marc, son originalité éclate au grand jour : à Marc qui, dans son article sur La nouvelle peinture, célèbre la rupture du Blauer Reiter avec la tradition académique et réaliste, Beckmann oppose son idéal classique et aristocratique de l'artiste et sa conception nietzschéenne de l'intemporalité des lois de l'art et de la morale ${ }^{3}$. Il défend une peinture à laquelle s'appliquent les trois définitions de l'histoire de la Seconde intempestive de Nietzsche. Selon le point de vue monumental, l'histoire « appartient à l'actif et au puissant, à celui qui participe à une grande lutte et qui, ayant besoin de maitres, d'exemples, de consolateurs, ne saurait les trouver parmi

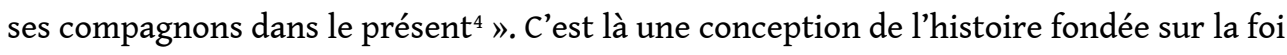
en l'humanité et de la fascination pour le sublime des grandes figures du passé, "l'intérêt pour ce qui est classique et rare dans les temps écoulés " ${ }^{5}$. S'opposant aux avant-gardes modernes, Beckmann se constitue de fait un panthéon personnel de peintres aussi divers que Tintoret, le Greco, Goya, Géricault, ou Delacroix car il découvre dans la peinture des maitres anciens une violence ou une force qui font écho au chaos de son temps. Il développe donc aussi un culte de l'histoire antiquaire: "L'histoire appartient donc en second lieu à celui qui conserve et vénère, à celui qui, 
avec fidélité et amour, tourne les regards vers l'endroit d'où il vient, où il s'est formé " ${ }^{6}$. Mais loin de momifier la vie en se détournant du présent, Beckmann, qui ne tire du passé que ce qui peut nourrir une peinture qui se fait intelligence du monde, pratique surtout l'histoire critique prônée par Nietzsche : une histoire servant la vie en tirant des leçons du passé utiles au présent grâce à la « force plastique » qui l'anime, ou en brisant le passé pour pouvoir renaitre: "Nous avons besoin de l'histoire pour vivre et pour agir, et non point pour nous détourner nonchalamment de la vie et de l'action [...]. Nous voulons servir l'histoire seulement en tant qu'elle sert la vie ${ }^{7}$ ». De fait, toute la vie de Beckmann balance entre la poursuite de l'héritage des maitres anciens et la quête d'un art propre, apte à répondre aux interrogations de la modernité et à servir la vie sans pour autant rompre totalement avec la tradition et deux de ses corollaires : la croyance au mythe et au sublime, qui permettent de dépasser la réalité en la transcendant.

3 Né en 1884 à Leipzig, Beckmann a trente ans lorsque la guerre éclate ; il opère alors un renversement copernicien en changeant radicalement de style. L'autre grande rupture de sa vie sera son exil volontaire à Amsterdam en 1933. Mais pour cet artiste éprouvé par une réalité hostile, qui dit s'être efforcé toute sa vie de devenir une "sorte de moi ", quête esthétique et identitaire sont inséparables : à travers ce moi qu'il poursuit dans journaux intimes et ses innombrables autoportraits, c'est un langage pictural au plus près de l'homme et de la vie qu'il cherche à élaborer. Un langage qui ne cesse d'osciller entre les deux pôles du beau et du laid, de l'espoir et de l'horreur, témoignant de l'ambiguïté fondamentale d'une vie envers laquelle Beckmann nourrit un rapport de fascination-répulsion, mais qui reste le fondement de son esthétique. Son Autoportrait avec les bulles de savon (vers 1900) qui le représente comme ligoté sur une chaise, mais le profil tendu vers un horizon symbole de liberté comme les bulles aériennes qui le surplombent, témoigne déjà de sa déchirure par trop humaine entre sa fascination pour la matière et sa volonté d'arracher son esprit à la pesanteur et à la laideur du monde. Tentons donc de suivre la quête du moi que Beckmann expose dans ses journaux et écrits personnels pour comprendre comment, chez cet être de métamorphoses, la réflexion sur la beauté et la laideur du monde est inséparable des variations de sa conception de l'homme et de l'histoire ${ }^{8}$.

\section{I - Du beau académique au beau pathétique}

4 À seize ans, Beckmann entre à l'École des Beaux-arts de Weimar : il se définit alors comme épigone du Tintoret, de Greco, Goya, Géricault et Delacroix et reconnaît chez des peintres contemporains tels Munch, Van Gogh et Cézanne la même capacité que leus aînés à exprimer le mystère de la vie ${ }^{9}$. Il réalise alors de nombreuses peintures de paysages et de nus comme Jeunes hommes au bord de la mer (1905), opposant aux baigneuses de Cézanne une vision plus virile et nordique où se mêlent tradition et modernité : la profondeur et l'immensité suggérées par l'océan à l'arrière-plan rappellent, note Spieler ${ }^{10}$, le Moine au bord de la mer (1808-1810) de Caspar David Friedrich, avec la différence toutefois que l'homme n'est pas écrasé par l'infini, mais semble le dominer par sa taille monumentale, sa beauté académique, sa gestuelle harmonieuse, son attitude contemplative et par la douceur pensive de ses traits. Jusqu'en 1906, les écrits et les œuvres de Beckmann témoignent encore de son attachement à cette beauté classique. Dans ses journaux de jeunesse, il écrit ce qu'il 
intitule des "notes pour une philosophie du beau» d'inspiration pleinement connaissable et compréhensible par l'homme qui en fait partie intégrante :

\section{Notes à propos de la philosophie de la beauté}

5 Le Tout considéré comme la forme primitive de la beauté éternelle. Saisir cette beauté par l'étude de ses reflets et de ses symboles. Chercher la preuve que la vie atteint le bonheur suprême quand elle ressemble le plus au calme et à la beauté éternels du Tout. témoignent de son hédonisme et de sa fascination pour le beau : en 1903, Beckmann évoque la beauté de la mort ${ }^{12}$, la " profusion de beauté $»^{13}$ d'un paysage montagnard, déclare que "l'humour fait partie intégrante de la beauté $~^{14}$ ou s'écrie : «Cherchez la beauté, battez-vous pour elle; luttez pour elle, elle vous donnera du plaisir. Ah, le plaisir ! Toute passion est beauté $\aleph^{15}$. Là où ça me plaît, je resterai. Et je rechercherai la beauté ${ }^{16}$. «Créer de nouvelles beautés. Vivre d'autres belles choses ${ }^{17}$. Le beau est aussi souvent associé à la passion amoureuse pour Minna Tube, rencontrée en 1902: «Elle est belle, pure et fine $»^{18}$, « Tout est clair et simple et il n'y a rien de laid ${ }^{19}$ ». Mais dès cette période, on sent également Beckmann attiré par une beauté sombre, dionysiaque, voire terrifiante. À Paris, il rencontre Munch, dont Le Cri (1895) trouve un écho saisissant dans l'Autoportrait de 1901. Le regard trouble et terrifié du sujet aux traits déformés par le cri d'angoisse qu'il profère témoigne de l'intranquillité qui sourd déjà chez Beckmann. Début septembre 1903, semblant réduire son culte du beau à une illusion de jeunesse, il note :

Rassure-toi, jeune homme, tu vas te résigner à ce sort comme à toutes les horreurs.

Où se situe la beauté totale ? Tu ne la trouveras jamais, espèce de petit-bourgeois stéréotypé, insensé et nostalgique ${ }^{20}$.

13 En avril 1904, il avoue avoir «beaucoup de mal à imaginer qu'il puisse y avoir encore quelque chose de beau dans ce monde ${ }^{21} »$. On trouve alors dans ses journaux quantité de notations oxymoriques associant beauté et laideur : "Je suis enfin rassuré. J’ai pu trouver quelque chose de laid dans un visage d'une grande ${ }^{22} » ;$ «C'est affreux. Toutes les beautés ${ }^{23}$ ». Il évoque également «l'horreur absolue ", «l'horrible beauté du sujet ${ }^{24}$ " de certains de ses propres tableaux De même, son $\mathrm{Nu}$ de la vieille femme (1905) au corps décharné et déformé par la vieillesse, isolée dans son chagrin, témoigne d'une volonté de dépouillement naturaliste opposée à l'«affectation impressionniste». On trouve donc dans les premières œuvres de Beckmann une première forme d'expression du 
sentiment de mélancolie qui imprègne tout le Journal et constitue le contrepoint à son enthousiasme pour le beau ${ }^{25}$.

En 1906 survient la première rupture capitale que représente la mort de sa mère et la découverte du pathétique de la vie et du destin de souffrance de l'humanité. À son retour à Berlin en 1907, le thème favori de Beckmann devient le drame humain: délaissant peu à peu les sujets traditionnels, il privilégie des sujets apocalyptiques. Il choisit les grands sujets de l'histoire religieuse et peint des peintures d'histoire de grand format, dans la tradition romantique d'un Géricault ou Delacroix: le Déluge (1908), la Scène de la destruction de Messine (1909), et le Naufrage du Titanic (1912). Le pathos reste très académique et Beckmann s'oppose à cette époque à la théorie picturale de l'art moderne défendue par Picasso, Braque, Matisse, Kandinsky, le Blauer Reiter ou Malevitch : il juge trop esthétique la réduction du tableau à son caractère plan et à son contenu formel. Beckmann, lui, soumet tout contenu au message à transmettre, même si, note Spieler, sa volonté d'exprimer ses émotions profondes et la grandiloquence des gestes et des formes donnent parfois l'impression de «cliché pathétique ${ }^{26}$. Dans une note de son journal du 9 janvier 1909 , il rejette désormais l'esthétisme purement formel de l'impressionnisme, trop décoratif à ses yeux et revendique, dans l'esprit de l'éloge nietzschéen de la vie dionysiaque, un art «plus brut, plus ordinaire, plus vulgaire, qui ne vit pas dans un monde de légendes, de rêves et de poésie ", un art qui " autorise un accès direct à ce qu'il y a de fécond, de commun, de grandiose et de banal dans la vie. Un art qui puisse être directement présent dans ce que la vie compte de plus réel ${ }^{27}$ : dès avant la guerre, il opte donc pour la peinture d'histoire pour lutter contre l'anonymat et l'indifférenciation modernes et pour restaurer une humanité véritable.

\section{II - L'« horrible beauté de la vie » : une esthétique nietzschéenne par delà le beau et le laid}

15 C'est véritablement avec la guerre que Beckmann découvre son moi propre. En 1912 déjà, il a été très ébranlé par sa visite chez Meidner dont il découvre les Paysages apocalyptiques. Mais c'est pendant la guerre qu'il médite sur la capacité de la peinture à exprimer le sens de l'histoire et découvre son propre style. Lui qui était le plus jeune espoir de la Sécession berlinoise rompt en 1913 ses liens avec elle et, en 1914, s'engage comme infirmer volontaire. Contrairement à Dix ou Grosz, qui s'enthousiasment pour la guerre, Beckmann prophétise la catastrophe et voit dans la guerre le plus grand malheur qui puisse frapper l'Allemagne. C'est en Belgique, où il soigne des soldats atteints du typhus et où il assiste à de nombreuses opérations, qu'il prend toute la mesure des atrocités de la guerre. Après s'être effondré psychologiquement et physiquement à la fin de l'été 1915, il est libéré de ses obligations militaires et s'établit à Francfort à partir de 1917.

Loin de se limiter pourtant à une expérience traumatique, la guerre constitue aux yeux de Beckmann un tournant esthétique : elle lui permet d'abandonner l'épigonalisme de ses débuts pour se tourner vers la vie et élaborer une nouvelle conception antiacadémique, nietzschéenne et vitale du beau. La guerre constitue en effet pour Beckmann une situation extrême, qui permet de pénétrer au plus profond de l'homme et d'exorciser ses passions les plus intimes. Mais elle implique une révolution du langage pictural. Le pathos historique de la première période de Beckmann était, note 
Spieler, « le langage formel d'une société féodale » qui s'effondre en 1914 : «les visions artistiques et allégoriques auxquelles se rattachaient les drames historiques de Beckmann se trouvaient balayées par la réalité avec une véhémence et une brutalité interdisant tout retour aux anciennes formes d'expression ${ }^{28}$ ». Dans une lettre du 11 mai 1915, Beckmann réduit donc tous ses travaux antérieurs à un simple apprentissage qui préludait aux véritables années de formation qu'il est en train de $v^{v i v r e}{ }^{29}$. Il écrit à sa femme qu'il expérimente dans la guerre « la maladie et la débauche, l'amour et la haine jusqu'à leurs dernières limites » et qu'il loue « cette ampleur, cette profondeur d'une inquiétante beauté ${ }^{30}$ qui surgit devant ses yeux :

17 Il ne s'agit pas pour moi de participer à cet événement en tant qu'historien, mais de m'habituer à cette chose qui est une manifestation de la vie telle que la maladie, l'amour et la volupté. Et tout comme personnellement, volontairement ou involontairement, je poursuis jusque dans ses derniers retranchements la peur de la maladie et de la luxure, l'amour et la haine, je tente désormais de faire la même chose avec la guerre. Tout est vie, merveilleusement variée et débordante d'inspiration. Je trouve partout les traces profondes de la beauté, dans le fait de souffrir et de supporter cet abominable destin ${ }^{31}$.

Dans son Nouveau Programme de 1914, Beckmann oppose l'art abstrait, décoratif et ornemental de la surface, la «matière décorative, plate et stylisante ", le " principe de Byzance et de Giotto ", à un art vital et expressif qui «se rapproche le plus possible de la vie par la spatialité et la plastique » et qui "privilégie la profondeur de champ ", chez Rembrandt, Tintoret, Goya, Courbet et le jeune Cézanne ${ }^{32}$.

Pour ce peintre qui se dit prêt à traverser "tous les cloaques du mondes", à subir "toutes les humiliations, tous les viols pour pouvoir peindre $»^{33}$, la guerre est l'expérience-limite idéale pour "prendre le pouls de la vie", "disséquer l'homme jusqu'aux nerfs ", "sonder le tréfonds de son âme ${ }^{34}$ ». L'effroi qu'il éprouve devant la souffrance dont il est témoin se double d'une fascination croissante pour l'intensité de vie qu'il découvre en même temps. Il écrit ainsi en 1915 :

J'espère devenir progressivement plus simple, plus concentré dans mon expression, mais jamais, je le sais, je ne renoncerai à la plénitude, à la rondeur, aux pulsions de

la vie, au contraire, j'aimerais les intensifier toujours davantage ${ }^{35}$.

Dans ces lettres du front, il développe une "esthétique macabre ${ }^{36} »$. On assiste également à une profonde mutation de son langage pictural : il abandonne les grands formats pour le dessin et la gravure, la mine tendre utilisée jusqu'en 1914 pour la plume de roseau, dure et pointue, qui permet un trait à la fois fin et nerveux, dont la précision quasi blessante évoque celle du scalpel. Ses modèles revendiqués sont alors les gothiques du Moyen Âge tardif, créateurs de ce que Beckmann appelle une "mystique virile », Matthias Grünewald, Gabriel Mälesskirchner ou le Greco. Stephan von Wiese diagnostique une "déstabilisation croissante de ses moyens d'expression, perceptible dans la désorganisation du système graphique, par des effets de dissolution, par l'agitation et la nervosité du trait, par le morcellement de la composition $»^{37}$ : celleci se disloque, le trait se fait plus nerveux, visages et objets semblent se fondre en un conglomérat de fragments et de lignes anguleuses et brisées. Dans L'Obus, pointe-sèche de 1914, qui marque la rupture avec le langage formel précédent, Beckmann fait éclater la perspective et exploser l'espace, figurant ainsi la folie de la destruction et donnant au dessin l'aspect convulsif des derniers soubresauts de la vie. Le dessin fait écho à la lettre du 4 mai 1915 où Beckmann évoque les blessures mortelles de deux soldats, « l'un 
tout pâle, brunâtre, avec une expression étrangement surnaturelle » et "l'autre avec un visage brutal, tout recouvert de sang sale avec une énorme plaie béante au menton et au cou, un abîme profond et sanguignolent ${ }^{38}$ ». Sylvie Ramond parle à ce propos de dédoublement, «un dédoublement à la fois ironique et absolument sérieux, voire arrogant ; une distance intériorisée par l'œuvre d'art, capable d'exorciser l'horreur de la vie et qui, selon lui, est le contenu même de l'art véritable ${ }^{39}$ ".

Le corps meurtri du Christ de la Descente de croix (1917), qui rappelle le visage tourmenté du Christ de Grünewald,puis Résurrection (1916-1918), fresque monumentale et inachevée, transposent en peinture le nouveau langage pictural de Beckmann. Renonçant à la perspective centrale au profit d'un kaléidoscope de formes fragmentées et de proportions déformées, de surfaces opposées les unes aux autres et d'obliques brisées, ce paysage apocalyptique est peuplé de figures décharnées dont les corps étirés évoquent le $\mathrm{Greco}^{40}$, mais dont les lignes brisées rappellent aussi Les Demoiselles d'Avignon de Picasso. Toutefois, Picasso donne une vie propre à une forme totalement émancipée du contenu, tandis que Beckmann la met au service de l'expression du chaos de la guerre : la "caricature mortifère du vivant $»^{41}$ de ce tableau mélancolique et désespéré dénie toute rédemption divine. Il y oppose la passion profane et la rédemption par l'art dans la Nuit. De ce tableau d'une violence absolue, qui applique à l'humanité tout entière le motif médiéval du Schmerzensmensch, où toutes les formes sont torturées et où le chaos des lignes et la saturation de l'espace témoignent de la perte de tout humanisme, Beckmann dit dans sa Confession créatrice qu'il est un "cauchemar qui a pris une ampleur métaphysique $"^{42}$. Dans une conversation avec Reinhard Piper, il développe à cette occasion sa nouvelle conception de la beauté, s'opposant à la beauté apollinienne des grecs qu'il dénonce comme illusoire ${ }^{43}$. Il défend une beauté sombre, née de l'expérience de la guerre conçue comme rupture avec la confusion et l'autosatisfaction bourgeoise ${ }^{44}$, mais aussi avec la croyance en un Dieu tout puissant. Mais elle n'implique pas pour autant le renoncement à toute dimension métaphysique, bien au contraire : c'est à l'artiste qu'il revient de la développer en partant du réel, mais en le transcendant ${ }^{45}$. L'art est le pilier de la nouvelle église qu'il entend fonder : c'est parce que la peinture lui permet de «trouver la vie objectivement laide $»^{46}$ qu'elle lui évite d'y sombrer en l'emprisonnant dans son tableau, en convertissant sa mélancolie en folie créatrice, en lui donnant forme, annihilant ainsi la puissance négative de l'objet recréé par la force de l'art.

C'est comme si la peinture se réduisait aux dimensions d'un voile invisible, mais pourtant bien réel. Un voile qui permet tout à la fois d'épuiser l'horreur de la vie en la fixant et en la portant à son intensité maximale, et d'attribuer à cet épuisement une valeur salvatrice pour les autres, puisque ce voile les en protège par son imperceptible épaisseur ${ }^{47}$.

Dans sa célèbre Confession créatrice de 1918, il affirme rechercher l'objectivité pour rendre ses visions intérieures ; contre la valorisation expressionniste de la construction intérieure et tournée vers l'abstraction, il défend une vision intérieure tournée vers la réalité pour «offrir aux hommes une image de leur destin » ressaisi par le prisme de l'art :

Je ne hais rien tant que la sentimentalité. Plus forte et plus intense devient ma volonté de fixer l'indicible de la vie, plus fortement, plus profondément et plus douloureusement je suis bouleversé par notre existence, plus ma bouche se ferme et plus ma volonté se tend dans le but de capturer ce monstre de vitalité aux convulsions terrifiantes, de le capturer, de l'abattre et de l'étrangler dans des 
surfaces et des lignes nettes plus transparentes que du verre. [...] Une main peinte ou dessinée, un visage ricanant ou pleurant, telle est ma profession de foi ; si j'ai ressenti quelque chose de la vie, cela se trouve là. [...] Il nous faut livrer nos cœurs et nos nerfs à l'épouvantable cri de détresse des pauvres gens trompés. [...] Pouvoir offrir aux gens une image de leur destin, on ne peut le faire que si on les aime ${ }^{48}$.

Dans les années vingt, Beckmann s'intègre au monde bourgeois, parvient de nouveau à vendre des tableaux à Francfort et, après avoir divorcé de Minna Tube, épouse Quappi von Kaulbach en 1925 : cette décennie est celle de l'harmonie et de l'analyse plus sereine du monde. Le contenu des œuvres change alors : las de se concentrer sur la violence de la vie, Beckmann, rejetant l'utopie édénique des expressionnistes et l'utopie techniciste des futuristes, se tourne vers le concret. Il lui rend hommage dans ses nombreuses natures mortes et paysages qui, en vertu de son exigence d'objectivité transcendentale, tendent à emprisonner le réel dans des lignes claires, des plans nets, des images claires et distinctes. Dans son Autoportrait en clown (1921), où il exhibe son bras droit comme un malade montrerait son corps guéri des stigmates de la maladie, les traits ne sont plus déformés ni le visage exsangue comme dans l'Autoportrait au foulard rouge de 1916, mais ronds, apaisés. Beckmann donne désormais priorité à la forme, à la transposition objective des visions intérieures. Mais cette prédilection pour ce qui est caché l'amène à se détacher progressivement de l'apparence extérieure des choses pour se tourner vers leur dimension mystique et mythique, sans pour autant se détourner de la réalité. En 1922, il évoque dans le Kunstblatt sa métaphysique de l'objectivité : il s'agit de chercher à partir de la réalité donnée le chemin de l'invisible, de rendre l'invisible visible en exorcisant la magie de la réalité. Beckmann utilise alors des moyens abstraits en donnant un caractère autonome au caractère plan de la couleur ; mais il les met au service d'un art figuratif dont l'originalité atteste de la toute puissance du créateur. Il 
développe encore plus sa métaphysique de l'artiste dans L'artiste dans l'état (1927) où il exhorte son époque à la "maîtrise élégante de la métaphysique " ${ }^{52}$. De même qu'en 1922 il avait distingué le moi moderne qui doit se satisfaire des vicissitudes de l'histoire, du moi lyrique incarnation de l'artiste, régressif, monomaniaque du mythe, il évoque en se référant à Schopenhauer, son second génie tutélaire depuis 1922, le moi transcendental comme moi véritable : l'artiste est Dieu, il est le véritable créateur d'un monde qui n'existait pas avant lui et comme tout homme, il doit être porté par la seule idée de la responsabilité de soi par rapport à l'infini :

L'artiste, au sens actuel du terme, est le créateur conscient de l'idée transcendante. [...] La nouvelle idée que l'artiste, et avec lui en même temps toute l'humanité, doit engendrer, est celle de l'auto-responsabilité. L'autonomie par rapport à l'infini. La solution de l'énigme mystique de l'équilibre, la déification définitive de l'homme..., tel est le but. [...] L'art est le miroir de Dieu qui est l'humanités.

Dans les années trente, Beckmann se tourne vers la mythologie, l'ésotérisme et le symbolisme: il lit les textes de Jung sur la relation entre la psyché individuelle et l'inconscient collectif, s'intéresse à l'exégèse des textes sacrés et découvre Helena Blavatsky. Cette fondatrice de la théosophie propose, dans sa Doctrine secrète (1932), une synthèse des philosophies ésotériques indienne et tibétaine. C'est à elle que Mondrian, Kupka et Kandinsky empruntent le principe de perfectibilité. Mais elle apporte surtout à Beckmann un réservoir de symboles et de mythes et une tendance à faire fusionner rêve, fantasmagorie et réalité.

Cherchant des archétypes éternels de la vie humaine, l'artiste les trouve dans le mythe. Mais loin de constituer l'utopie d'un monde idéal, le mythe est celui de l'éternel destin auquel l'homme est enchaîné. Au début des années trente, on assiste donc à un nouveau changement: Beckmann dénonce comme utopique son postulat antérieur de la liberté humaine et ne croit plus à la possibilité de transformer les forces négatives en forces positives. La vie humaine est un combat perpétuel, notamment dans les trois sphères désormais centrales pour l'artiste : la connaissance, la sexualité, la faute. C'est là le sujet du triptyque Le Départ (1932-1935) dont le volet gauche met en scène dans le rôle du bourreau torturant un homme et s'apprêtant à violer une femme un pêcheur apparemment inoffensif. Il dénonce la passivité du peuple allemand face au régime de terreur nazie qui le condamne à l'exil à Amsterdam, puis aux Etats-Unis. Le départ de Beckmann a en effet lieu aussitôt après qu'il a entendu à la radio l'allocution prononcée par Hitler pour l'ouverture de la «Grande exposition d'art allemand » et où Hitler fustige les tenants de l'« art dégénéré » et la prétendue laideur de leurs productions :

Ces êtres difformes et demeurés [...]. Voilà ce que ces redoutables dilettantes d'aujourd'hui osent nous présenter comme l'art de notre époque [...]. Ou bien ces soi-disant «artistes » voient vraiment les choses comme cela et croient donc à ce qu'ils représentent, et il faudrait alors examiner si leur déficience oculaire est de nature mécanique ou héréditaire. [...] Ou bien ils ne croient pas eux-mêmes à la réalité de telles impressions mais ils s'efforcent pour d'autres raisons d'importuner la nation avec de tels mensonges et leur comportement relève alors du droit pénal ${ }^{54}$.

Beckmann voit aussi dans cet exil la possibilité de se libérer du poids de l'existence passée. C'est le sens du symbole nietzschéen de l'enfant du panneau central du Départ: il contemple les rivages plus heureux vers lesquels il va être porté avec le roi et la reine. Mais la symbolique du panneau de droite est plus ambiguë; si le poisson est toujours symbole de fertilité et de fusion érotique chez le dernier Beckmann, les liens qui emprisonnent la famille arrivée en terre d'exil lui ôtent toute liberté de mouvement et 
contredisent la liberté promise par le panneau central. Isolé car condamné à l'exil et à l'émigration intérieure et contraint de devenir un artiste anonyme à Amsterdam, Beckmann retombe de fait dans les années 1937 à 1947 dans un mépris et une négation absolus du monde annoncés dès 1945 dans son Journal :

Jusqu'ici, il s'agit encore et toujours de définir le Bien et le Mal, le Beau et le Laid en fonction de ses forces. Lorsqu'on constate l'insuffisance de ces stimuli est établie, subsiste néanmoins le danger de la négation ${ }^{55}$.

31 Mais en 1947, la vitalité et la superficialité des États Unis ainsi que le succès dont il y jouit et qu'il se plaît à évoquer dans ses journaux l'amènent à tempérer un peu son pessimisme et à lui opposer la conviction que vie, mort et salut forment une unité indissoluble : l'homme est condamné à vivre sur terre, avec ses horreurs et ses beautés, tel l'Icare de la Chute (1950), entraîné malgré lui vers le monde terrestre, sa seule patrie. Il s'agit désormais pour le peintre de substituer à la passivité de la mélancolie l'élan créateur qui permet à la fois d'intensifier l'horreur en la répétant sur la toile et de la dénégativer en l'objectivant dans une démarche qu'il nomme tantôt métaphysique, tantôt transcendentale: "vaincre le laid par une laideur accrue, parachever avec la violence propre à l'esprit et à l'art la violence intrinsèque du réel ${ }^{56} »$. Il lui faut détruire l'objet, non en le supprimant comme les expressionnistes, mais au contraire en le maintenant, en rendant le malheur et la laideur objectifs, conscients. Il s'oppose aussi à l'objectivité non métaphysique de Grosz ou de Dix qui recourent à la déformation et à la laideur pour dénoncer une réalité sociale à leurs yeux intolérable; le propos de Beckmann, lui, est métaphysique: il s'agit de proposer une rédemption, même provisoire, en présentant aux hommes une « image de leur destin » : pour cet artiste fasciné par les miroirs, l'art est donc un miroir de l'humain trop humain, mais un miroir qui n'invite pas à contempler l'image figée des laideurs de l'existence, mais à la dépasser, dans un mouvement de survie. Car il s'agit bien d'une esthétique de l'intranquillité et du dévoilement, où le pessimisme le dispute à l'humanisme et lutte à la fois contre la laideur de la vie et pour la beauté d'une vie transmuée par l'art. Mais transcender le monde lui permet moins d'en retrouver la beauté, que d'en restaurer la sublime unité par le mythe. Il choisit donc la voie du symbolisme qu'il oppose à l'allégorie qui fige le sens et le fait préexister à l'œuvre ; le sens de l'œuvre naît pour lui de l'image puisque sa métaphysique est ancrée dans la vie indistincte du rêve :

À partir d'une réalité donnée, je cherche les ponts vers l'invisible, à l'instar du célèbre cabaliste qui a affirmé : «si tu veux appréhender l'invisible, pénètre aussi profond que tu le peux dans le visible ». Pour moi, il s'agit toujours de saisir le magie de la réalité et de la transcrire en peinture. De rendre l'invisible visible au moyen de la réalité ${ }^{57}$.

Beckmann retourne ainsi aux origines de l'art romantique. En 1943, il écrit d'ailleurs à Lily von Schnitzler que, s'inspirant de Jean Paul, il renoue avec l'inspiration romantique: il confond à dessein rêve et poésie en développant une symbolique subjective fondée sur l'ambivalence entre réalisme et idéalisme subjectif. Ce que Beckmann appelle de ses vœux dans son Journal de 1945, c'est la "sublimation de l'aspect visuel en un autre niveau d'existence" pour lutter contre la "désillusion absolue " qui menace son époque ${ }^{58}$. L'art ne doit pas être décoratif, mais servir la connaissance mystique du mystère du monde et se faire promesse de salut. Beckmann développe ainsi une dialectique opposant la mélancolie et le pessimisme face au chaos du monde moderne à l'aspiration à l'unité mythique du monde. C'est par l'art qu'il 
parvient à une synthèse entre sa conscience moderne et sa fidélité à l'idéalisme ancien, entre le noir et le blanc, le laid et le beau :

C'est le rêve de bien des gens de ne voir que le blanc (ce qui est objectivement beau) ou le noir (le laid, le négatif)... Je ne puis faire autrement que me réaliser dans les deux. Ce n'est que dans les deux à la fois, le blanc et le noir, que je saisis Dieu dans son unité, Dieu ne cessant de récréer le spectacle toujours changeant du monde ${ }^{59}$.

Les héros de son ultime triptyque des Argonautes sont les artistes, qui ne cherchent pas la Toison d'or, mais le sens des choses et parviennent par l'art à montrer le chemin vers la vérité, l'essence intime du monde. C'est là le sens du panneau central dominé par deux jeunes hommes, Orphée au centre, Jason à droite. Représentés de dos, dans la même attitude que les Jeunes hommes au bord de la mer, mais avec des corps plus lourds et moins gracieux, ils illustrent comme eux l'association néoplatonicienne du beau et du bien tout en y ajoutant l'insistance moderne sur la lourdeur de la matière. La dynamique de leurs gestes témoigne aussi d'une assurance qui semblait faire défaut aux Jeunes hommes perplexes ou contemplatifs. Enfin, à la perspective horizontale du tableau de 1905 s'ajoute ici une dimension de verticalité et de profondeur. Les deux jeunes gens s'apprêtent en effet à gravir l'échelle que leur tend le peintre du panneau de gauche qui incarne Beckmann lui-même. Devenu dans le panneau central passeur entre l'univers physique et métaphysique, il leur permet d'accéder au nouveau paradis de l'art et de la musique figuré dans le panneau de droite. Dans cette œuvre-testament, le peintre fait de l'art la seule création humaine capable de faire accéder l'homme à une forme de sublime métaphysique transcendant la laideur et la souffrance du monde: l'art est le seul refuge de la liberté et du rêve. Lui seul permet de se libérer du voile trompeur des apparences et d'accéder à l'Être véritable. Beckmann illustre par là l'esthétique idéaliste de Schopenhauer tout en annonçant l'esthétique négative prônée par Adorno, qui dénonce la laideur du monde en recourant à la déformation et à la dissonance, mais qui maintient l'horizon d'une réconciliation, signe de la persistance de l'humanisme pourtant mis à mal par Auschwitz et par l'« industrie culturelle». Beckmann, comme Adorno, illustrent ici un changement de paradigme : ils renoncent à une beauté classique et académique dénoncée comme illusoire et décorative, fruit d'une culture dépassée qui exigeait du spectateur une passivité et une sérénité que les troubles de l'Histoire ont rendus impossibles:

Le laid dans l'art est d'abord la revendication d'une sensibilité saturée d'un classicisme qui ne trouve plus de résonances dans des époques saturées par les turbulences de l'Histoire : la répétition de la beauté canonique à laquelle conduit l'académisme, finit par lasser. En réaction à ce qui est ressenti comme fade et monotone, la recherche de la déformation [...] a pour effet de donner à l'œuvre la saveur et la diversité de la vie ${ }^{60}$.

Beckmann comme Adorno postulent un art autonome, qui serve «la connaissance, et non le divertissement $\aleph^{61}$; ils démontrent la nécessité d'intégrer la laideur, la noirceur et la négativité à un art qui se fait désormais résistance et dénonciation critique des laideurs de l'histoire: «l'art doit dénoncer, par le laid, le monde qui le crée et le reproduit à son image", dit Adorno ${ }^{62}$. Mais Beckmann ne prône pas pour autant le renoncement à l'utopie; celle-ci réclame l'inquiétude et l'arrachement propres au sublime romantique et moderne, qui est d'abord «ce dans quoi le monde se déstabilise ", l'« irruption d'un hors-temps du trouble, du doute et de la solitude ${ }^{63}$. Le sublime "assigne une position, réveille une exigence et entraine un processus de conversion " ${ }^{64}$ à l'infini : sublime est la capacité de l'homme à s'élever au-dessus de sa condition, sublime est le pouvoir de l'art de libérer l'homme de ses entraves; sublime 
enfin est le geste salvateur du porteur d'échelle des Argonautes, lointain descendant de Schiller : seule la sphère esthétique permet à l'homme de transcender le divorce entre l'esprit et la matière, la forme et l'instinct, le blanc et le noir, le beau et le laid, pour se réconcilier pleinement avec lui-même et avec le monde.

\section{NOTES}

1. Propos d'Alfred Kubin cités par Reinhard Piper in: Mein Leben als Verleger, München, Piper, 1947, p. 469.

2. Philippe Dagen, préface aux Écrits de Beckmann, Paris, Ecole Nationale Supérieure des BeauxArts, 2002, p. 8.

3. «Die Gesetze der Kunst sind ewig und unveränderlich, wie das moralische Gesetz in uns. », in : M. Beckmann, Gedanken über zeitgemäße und unzeitgemäße Kunst, März 1912, in : Die Realität der Träume in den Bildern, Aufsätze und Vorträge aus Tagebüchern, Briefen, Gesprächen, 1903-1950, Leipzig, Reclam, 1987, S. 42.

4. Friedrich Nietzsche, Seconde considération intempestive, trad. H. Albert, Paris, GarnierFlammarion, 1988, p. 87 / Vom Nutzen und Nachteil der Historie für das Leben, Stuttgart, Reclam, 1970, S. 19: «Die Geschichte gehört vor allem dem Tätigen und Mächtigen, dem, der einen großen Kampf kämpft, der Vorbilder, Lehrer, Tröster braucht und sie unter seinen Genossen und in der Gegenwart nicht zu finden vermag. »

5. Ibid., p. 90/S. 23 : « Die Beschäftigung mit dem Klassischen und Seltenen früherer Zeiten. »

6. Ibid., p. 95/S. 27 : «Die Geschichte gehört also zweitens dem Bewahrenden und Verehrenden, dem, der mit Treue und Liebe dorthin zurückblickt, woher er kommt, wohin er geworden ist. ».

7. Ibid., p. 72/S. 3 : « Wir brauchen sie zum Leben und zur Tat, nicht zur bequemen Abkehr vom Leben und von der Tat [...]. Nur soweit die Historie dem Leben dient, wollen wir ihr dienen. »

8. On dispose actuellement en Allemagne d'une édition complète de la correspondance de Beckmann : Max Beckmann, Briefe, Bd. 1 : 1899-1925/Bd. 2 : 1925-1937/Bd. 3 : 1937-1950, Hrsg. von Klaus Gallwitz, Uwe M. Schneede, Stephan von Wiese, München, Piper, 1993-1996. Mais il n'existe à ce jour pas d'édition complète des journaux, qui sont édités en 5 volumes distincts, regroupés par périodes, et proposent souvent des extraits au lieu du texte intégral : Leben in Berlin; Tagebuch 1908-1909, Hrsg. Hans Hinkel, München, Piper, 1966-1983 ; Max Beckmann frühe Tagebücher 1903-1904 - 1912-1913, Hrsg. von Doris Schmidt, München, Piper, 1985 ; Tagebücher 1940-1950, gesammelt von Mathilde Quappi-Beckmann, Hrsg. von Erhard Göpel, München, Piper, 1955 ; Briefe im Kriege, gesammelt von Minna Beckmann Tube, Berlin, 1916 - 2. Auflage : München, Piper, 1955 ; Die Realität der Träume in den Bildern. Aufsätze und Vorträge. Aus Tagebüchern, Briefen, Gesprächen, 1903-1950, Hrsg. von Rudolf Pillep, Leipzig, 1987/München, Piper, 1990. C'est cette édition ainsi que celle des Frühe Tagebücher et des Briefe im Kriege que nous avons utilisée. En France, on ne dispose pas non plus d'édition intégrale des œuvres de Beckmann. L'édition la plus complète est l'édition très récente des Ecrits de Max Beckmann, traduction Thomas de Kayser, textes réunis et présentés par Barbara Stehlé-Akhtar, Paris, École Nationale Supérieure des Beaux-arts, 2002. Sans prétendre à l'exhaustivité, elle propose une sélection des Frühe Tagebücher dans l'édition de Doris Schmidt, des Lettres de guerre, des conférences de Beckmann et des Journaux abrégés 1940-1950. Nous en livrons la traduction et la modifions en cas d'inexactitude par rapport à l'original. 
9. Max Beckmann, Die Realität der Träume in den Bildern, Aufsätze und Vorträge aus Tagebüchern, Briefen, Gesprächen, 1903-1950, Leipzig, Reclam, 1987, S. 39 : «Ich selbst verehre in Cézanne ein Genie. Er konnte durch seine Bilder auf eine neue Weise die mysteriöse Weltempfindung ausdrücken, die vor ihm schon Tintoretto, Greco, Goya, Géricault und Delacroix beseelte. »

10. Reinhard Spieler, Max Beckmann, 1884-1950. L'apparition du mythe, Köln, Taschen, 1995.

11. Max Beckmann, Journal, 23.8.1903, Écrits, p. 38/Frühe Tagebücher, Hsrg. und kommentiert von Doris Schmidt, München, Zürich, Piper, 1985, S.17 : « Notizen zur Philosophie der Schönheit. Das All betrachtet als die Grundform der ewigen Schönheit. Das Erfassen dieser Schönheit durch Studium seiner Spiegelungen und Gleichnisse. Beweis zu suchen dafür daß das Leben am unglücklichsten ist, wenn es am ähnlichen die ewige Ruhe und Schönheit des Alls widerspiegelt. [...] Grundbedingung zur möglichst reinen und klaren Erkenntnis der Schönheit : 1) die feste und verstandene Überzeugung ein Teil derselben zu sein. 2) das All und also auch uns ruhig empirisch auffassen [...] 3) das völlig erfasste und verstandene der Schönheit je nach seiner Art uns mitzuteilen. Ziel der Erkenntnis, die Schönheit zu suchen, bis sie vor uns steht klar und ewig. Die selbstverständliche Veredlung, die auch der niedrigststehende Mensch durch die Erkenntnis einer so geringen Schönheit erleben wird. »

12. Ibid., p. 38/S. 18: «Der Tod ein sichtbarer Beweis für den Pulsschlag des Alls... und die Schönheit des Todes».

13. Ibid., p. 39/S. 18 : «Eine so überreiche Schönheit».

14. Ibid., p. 38-39/S. 19 : «Ich glaube, daß der Humor einen Teil der Schönheit ausmacht».

15. Ibid., 30.8. 1903, p. 42/S. 22 : «Sucht die Schönheit kämpft um sie ringt um sie [...] Sie wird auch Genuß geben. Ach Genuß. Alle Leidenschaft ist Schönheit.»

16. Ibid., 15.1. 1904, p. 74/S. 79 : «Wo es mir gefällt, da werde ich bleiben. Und die Schönheit suchen.»

17. Ibid., p. 80/S. 91 : «Neue Schönheiten schaffen. Neue Schönheiten erleben.»

18. Ibid., 3 août 1903, p. 44/S. 25 : «Sie ist so schön und rein und fein.»

19. Ibid., 3 septembre 1903, p. 44/S. 26 : «Es ist alles klar und einfach und nicht das geringste Hässliche dabei.»

20. Ibid., 6 septembre 1903, p. 46/S. 28 : «Schönheit ist mir nötiger als Luft du Licht. Beruhige dich mein Junge, auch hierin wirst du dich fügen, wie in all das andere Ekle auch. Wo ist denn ganze Schönheit. Du wirst sie nicht finden, du thörichter schablonenhafter Sehnsuchtsmeyer.»

21. Ibid., 7 avril 1904, p. 87/S. 100 : «Eigentlich kann ich mir kaum vorstellen, daß es irgendetwas Schönes in der Welt gibt.»

22. Journal, 9.12.1903, Écrits, p.65/S. 54 : «Endlich bin ich beruhigt, an einem sehr schönen Gesicht etwas Hässliches gefunden zu haben.»

23. Ibid., 7.4.1904, p. 86/S. 98 : «Es ist schrecklich. Alle Schönheiten.»

24. Ibid., 21.1.1909, p. 111/Max Beckmann. Leben in Berlin. Tagebuch 1908-1909, kommentiert und herausgegeben von Hans Kinkel, München, Zürich, Piper, 1983-1984, S. 31-32 : «(die) grauenhafte Schönheit des Sujets.»

25. On note à ce propos une profonde affinité entre les journaux de Beckmann et Delacroix, dont l'esthétique est également «marquée par le vacillement de l'ancien système pictural» (classique) et qui pratique comme Beckmann une "écriture de l'ébranlement ", du doute et de l'enfermement dans la mélancolie née de la ruine d'un idéal : « la pratique du journal entretient le mal sans le guérir : connaître et affronter la douleur n'est la promesse d'aucune délivrance, d'aucun deuil. »Cf. Anne Larue, Romantisme et mélancolie. Le Journal de Delacroix, Paris, Champion, 1998, p. 19 et 138.

26. Reinhard Spieler, L'Apparition du mythe, p. 23.

27. Max Beckmann, Journal du 9.1.1909, Ecrits, p. 104 / Tagebücher, in : Die Realität..., S.28 : «zu dekorativ.». «Mein Herz schlägt mehr und mehr nach einer roheren gewöhnlicheren vulgäreren Kunst, die nicht verträumte Märchenstimmungen lebt zwischen Poesien, sondern dem 
Furchtbaren, Gemeinen, Großartigen, Gewöhnlichen, Grotesk-Banalen im Leben direkten Eingang gewährt. Eine Kunst, die uns im Realsten des Lebens immer unmittelbar gegenwärtig sein kann.» 28. Reinhard Spieler, Max Beckmann, 1884-1950. L'apparition du mythe, p. 28.

29. Ibid., S. 68 ff. : «Es ist mir ganz recht, dass Krieg ist. Was ich bis jetzt gemacht habe, war alles noch Lehrjahre, ich lerne immer noch und erweitere mich.»

30. Beckmann, lettre du 11 octobre 1914, Écrits, p. 135 (trad. revue par nous-même)/Die Realität..., S. 51 : «Ach, diese Weite und unheimlich schöne Tiefe!»

31. Beckmann, lettre du 24.5. 1915, Écrits, p. 163/Briefe im Kriege..., München, Zürich, Piper, 1993, S. 75 : «Es handelt sich nicht darum, dass ich gewissermaßen als Historiker diese Angelegenheit mitmache, sondern, dass ich mich selbst in dieser Sache einlebe, die an sich eine Erscheinungsform des Lebens ist, wie Krankheit, Liebe oder Wollust. Und genau so, wie ich ungewollt und gewollt der Angst der Krankheit und der Wollust, Liebe und Hass bis zu ihren äußersten Grenzen nachgehe - nun, so versuche ich es eben mit dem Kriege. Alles ist leben, wunderbar abwechslungsvoll und überreich an Einfällen. Überall finde ich tiefe Linien der Schönheit im Leiden und ertrage dieses schaurigen Schicksals.»

32. Beckmann, Réponse à l'enquête "Le nouveau programme " de la revue Kunst und Künstler, Écrits, p. 126/Beitrag zu : Das neue Programm (1914), Die Realität der Träume, S. 43 : «Es gibt meiner Meinung nach zwei Richtungen in der Kunst. Eine [...] ist die flache und stilisierend dekorative, die andere ist die raumtiefe Kunst. Das Prinzip Byzanz, Giotto gegen das Prinzip Rembrandt, Tintoretto, Goya, Courbet und den frühen Cézanne. Das eine sucht die ganze Wirkung in der Fläche, ist also abstrakt und dekorativ, das andere sucht mit räumlicher und plastischer Form dem Leben unmittelbar nahezukommen. Plastik und Raumwirkung in der Malerei braucht nun aber noch lange kein naturalistischer Effekt zu sein. Es kommt auf die Kraft der Darstellung und des persönlichen Stils an (Rembrandt, Cézanne, Goya).»

33. Beckmann, Lettres de guerre, 21.4.1915, p. 151 / Briefe im Kriege, in : Die Realität..., S. 64 : «Ich würde mich durch sämtliche Kloaken der Welt, durch sämtliche Erniedrigungen und Schändungen hindurchwinden, um zu malen.»

34. Reinhard Spieler, "La rupture de la Première Guerre mondiale ", Catalogue d'exposition Beckmann, Paris, Editions du Centre Pompidou, 2002, p. 119.

35. Beckmann, lettre du 16 mars 1915, Écrits, p. 139-140/Briefe, S. 53 : «Ich hoffe allmählich immer einfacher zu werden, immer konzentrierter im Ausdruck, aber niemals, das weiss ich, werde ich das Volle, das Runde, das lebendig Pulsierende aufgeben, im Gegenteil. Ich möchte es immer mehr steigern.»

36. Reinhard Spieler, «La rupture de la Première Guerre mondiale », Catalogue d'exposition Beckmann, Paris, Éditions du Centre Pompidou, 2004, p. 119.

37. Stephan von Wiese, Max Beckmanns zeichnerisches Werk 1903-1925, Düsseldorf, Droste, 1978, cité par R. Spieler, op. cit., p. 120.

38. Beckmann, 4.5. 1915, Ecrits, p. 156-157/Briefe im Kriege, Die Realität der Träume..., S. 68 : «Der eine ganz fahl braunweiss mit merkwürdig überirdischem Ausdruck. Der andere ein brutales Gesicht, ganz mit schmutzigem Blut bedeckt, mit einer riesigen klaffenden Wunde am untern Gesicht und am Hals, ein Granat wie ein tiefer, blutiger Abgrund.»

39. Syvie Ramond, « Maîtres anciens, maîtres inactuels », Catalogue de l'exposition Beckmann, p. 133.

40. Ibid., p.132: Sylvie Ramond note que dès avant 1914, le Greco jouit d'un immense engouement en Allemagne. C'est avant tout son Laocoon présenté à l'Alte Pinakothek en 1910 et 1913 qui a frappé les esprits.

41. Maria Stavrinaki, «La destruction en acte. Beckmann, la guerre et le salut », Catalogue de l'exposition Beckmann, p. 145.

42. Gespräch mit Reinhard Piper, Die Realität der Träume..., S. 92 : «Es war ein Alpdruck, der sich ins Metaphysische steigerte.» 
43. Gespräch mit Reinhard Piper, Die Realität der Träume..., S. 94 : «Die Griechen sind mir fatal. Eigentlich war die ganze schöne Götterwelt, die sie sich vorgemacht haben, doch ein bewusster Schwindel.»

44. Beckmann, Gespräch mit Reinhard Piper, Juli 1919, Die Realität der Träume..., S. 94 : «Es ist doch eigentlich viel schöner, als vor dem Krieg, wo alles stumpf und satt war.»

45. Beckmann, Die Realität der Träume..., S. 92: «Man soll das Gegenständliche über dem Metaphysischen vergessen. Es soll durch das Metaphysische überwunden werden. Man soll nur die Schönheit sehn, wie auch ein Trauermarsch schön ist.»

46. Maria Stavrinaki, op. cit., p. 146.

47. Maria Stavrinaki, op. cit., p. 146.

48. Beckmann, Une Confession. Contribution au thème "Confession créatrice " dans la série «Tribune de l'art et du temps ", in : Écrits, p. 192 (trad. revue par nous-même) / Ein Bekenntnis. Beitrag zu : Schöpferische Konfession (1920), in : Die Realität..., S. 89-90 : «Nichts hasse ich so wie Sentimentalität. Je stärker und intensiver mein Wille wird, die unsagbaren Dinge des Lebens festzuhalten, je schwerer und tieferer die Erschütterung über unser Dasein in mir brennt, um so verschlossener wird mein Mund, um so kälter mein Wille, diese schaurig zuckende Monstrum von Vitalität zu packen und glasklarer scharfe Linien und Flächen einzusperren, niederzudrücken, zu erwürgen [...]. Eine gemalte oder gezeichnete Hand, ein grinsendes oder weinendes Gesicht, das ist mein Glaubensbekenntnis ; wenn ich etwas vom Leben gefühlt habe, so steht es so da drin [...]. Unser Herz und unsere Nerven müssen wir preisgaben dem schaurigen Schmerzensgeschrei der armen getäuschten Menschen. [...] Daß wir den Menschen ein Bild ihres Schicksals geben, und das kann man nur, wenn man sie liebt.»

49. Beckmann, Une confession (1918), p. 194 / Ein Bekenntnis. Beitrag zu Schöpferische Konfession (1920), 1918, S. 91 : «Aus einer gedanklosen Imitation des Sichtbaren aus einer schwächlich archaistischen Entartung in leeren Dekorationen und aus einer falschen und sentimentalen Geschwulstmystik heraus werden wir hoffentlich zu der transzendenten Sachlichkeit kommen, die aus einer tieferen Liebe zur Natur und den Menschen hervorgehen kann.»

50. Ibid., p. 194 (trad. revue par nous-même)/S. 91 : «Einmal Gebäude zu machen zusammen mit meinen Bildern. Einen Turm zu bauen, in dem die Menschen all ihre Wut und Verzweiflung, all ihre arme Hoffnung Freude und wilde Sehnsucht ausschreien konnten. Eine neue Kirche.»

51. Nietzsche, Nachgelassene Fragmente, XIV, § 174, Kritische Studienausgabe, Hsg. Colli-Montinari, Berlin, de Gruyter, 1986, S. 453-454: «Sodann soll die Kunst alles Hässliche verbergen oder umdeuten... Sie soll so namentlich in Hinsicht auf die Leidenschaften und seelischen Schmerzen und Ängste verfahren und unvermeidlich oder unüberwindlich Hässlichen das Bedeutende durchschimmern lassen.»

52. Beckmann, L'Artiste dans l'État, article pour l'Europäische Revue, juillet 1927, in : Écrits, p. 207 / Der Künstler im Staat, in: Die Realität..., S. 119: «Eine elegante Beherrschung des Metaphysischen».

53. Beckmann, L'Artiste dans l'État, article pour l'Europäische Revue, juillet 1927, Francfort-sur-leMain, Écrits, p. 206-207 (trad. revue par nous-même)/Der Künstler im Staat, in : Die Realität der Träume..., S. 117-118: «Der Künstler im neuen Sinn der Zeit ist der bewusste Former der transzendenten Idee [...]. Die neue Idee, die der Künstler und mit ihm zu gleicher Zeit die Menschheit zu formen hat, ist Selbstverantwortung. Autonomie im Verhältnis zur Unendlichkeit. Endültige Vergottung des Menschen ist das Ziel. [...] Die Kunst ist der Spiegel Gottes, der die Menschheit ist.»

54. Cité in R Spieler, L'Apparition du mythe, p. 123.

55. Beckmann, Journal du 28 octobre 1945, Écrits, p. 317 (trad. revue par nous-même) / Die Realität ..., S. 262-263 : «Bis dahin handelt es sich wohl darum, Böses und Gutes, Häßliches und Schönes, nach Maßstab der Kräfte, immer wieder festzustellen. 
Die Gefahr der Negation, bei der Feststellung der bedenkenden Unzulänglichkeit der gegebenen Reizmittel bleibt aber bestehen.»

56. Maria Stavrinaki, op. cit., p. 146.

57. Beckmann, À propos de ma peinture, 21 juillet 1938, Londres, in : Écrits, p. 215 / Die Realität..., S. 134-135 : «Ich suche aus der gegebenen Gegenwart die Brücke zum Unsichtbaren... ähnlich wie ein berühmter Kabbalist es einmal gesagt hat: "Willst du das Unsichtbare fassen, dringe so tief du kannst ein - in das Sichtbare." Es handelt sich für mich immer wieder darum, die Magie der Realität zu erfassen, und diese Realität in meine Malerei zu übersetzen. Das Unsichtbare sichtbar machen durch die Realität.»

58. Beckmann, Journal, 28 octobre 1945, Écrits, p. 317 (trad. revue par nous-même) / Die Realität..., S. 262-263: «Es kann sich also höchstens um eine Veränderung und hoffentlich höhere Steigerung des visuellen Aspektes in einem anderen Dasein handen [...]. Unser Zeitalter wohl nicht anders zu bezeichnen als "Zeitalter der absoluten Desillusion.»

59. Beckmann, À propos de ma peinture, 21 juillet 1938, Londres, in : Écrits, p. 217 (trad. revue par nous-même) / Die Realität..., S. 136 : «Trotzdem ist es doch der Traum vieler, nur das Weiße (nur das gegenständlich Schöne) oder nur das Schwarze (das Hässliche und Verneinende) sehen zu wollen... Ich kann nicht anders als mich in Beiden zu realisieren. Nur in beiden, Schwarz und Weiß, sehe ich wirklich Gott als eine Einheit, wie er sich als großes ewig wechselndes Welttheater immer wieder neu gestaltet.»

60. Michel Ribon, Esthétique de l'effacement. Essai sur l'art, Paris, L'Harmattan, 2005, p. 115.

61. Beckmann, À propos de ma peinture, in : Écrits, p. 217 / Über meine Malerei, in : Die Realität..., S. 136 : «Kunst dient der Erkenntnis, nicht der Unterhaltung.»

62. Adorno, Théorie esthétique, trad. M. Jimenez, Paris, Klincksieck, 1995, p. 78 / Ästhetische Theorie, Frankfurt, Suhrkamp, S. 78-79: «Kunst muß im Hässlichen die Welt denunzieren, die es nach ihrem Bild schafft und reproduziert.»

63. Baldine Saint-Girons, Fiat lux. Une philosophie du sublime, Paris, Quai Voltaire, 1993, p. 19-29.

64. Ibid., p. 30.

\section{RÉSUMÉS}

Partant du constat de l'oscillation constante de Max Beckmann entre espoir et mélancolie, beauté et laideur, cette étude évoque les trois grandes étapes qui ont marqué le cheminement esthétique de ce peintre inclassable, épigone à ses débuts, adversaire des avant-gardes futuriste et expressionniste, et qui a lutté toute sa vie pour accéder à une identité et à un style indissociables de sa conception de l'homme et de l'histoire. Jusqu'à la Première Guerre mondiale, Beckmann cultive une beauté académique dans la tradition néoplatonicienne de l'association du beau et du bien. Mais on décèle déjà des germes d'une intranquillité et d'une mélancolie qui se révèlent pleinement en 1914. La guerre marque en effet une seconde rupture qui amène Beckmann à se détourner du beau apollinien pour rechercher dans le choc et la violence du réel l'« effroyable beauté » dionysiaque et l'intensité vitale qui lui permet d'opérer la catharsis de l'horreur de la vie transmuée en art. Enfin, la troisième période, à partir des années 20, marque le dépassement métaphysique du dualisme entre beauté et laideur: Beckmann développe sa métaphysique de l'objectivité par laquelle il transcende le monde, découvre l'invisible sous-jacent dans le visible et parvient à restaurer l'unité mythique du monde, réconcilié avec le sujet par le prisme de l'art. Cet être de métamorphoses illustre donc le passage d'une conception encore académique du beau à 
un art pathétique, qui inclut le laid, jusqu'à un art métaphysique, qui transcende l'opposition entre beauté et laideur en les intégrant à une esthétique du sublime moderne.

Ausgehend von der Feststellung des ständigen Schwankens Max Beckmanns zwischen Hoffnung und Melancholie, Schönheit und Hässlichkeit, ruft folgende Studie die drei wichtigen Stufen der ästhetischen Entwicklung jenes unklassifizierbaren Malers hervor, der anfangs Epigone und Gegner der futuristischen und expressionistischen Avantgarden war, und der sein ganzes Leben lang kämpfte, um eine von seiner Menschen- und Geschichtsauffassung unzertrennliche persönliche und stilistische Identität zu gewinnen. Bis zum ersten Weltkrieg pflegt Beckmann eine akademische Schönheit in der neuplatonischen Tradition der Koppelung des Schönen und des Guten. Doch sind schon damals Keime einer Unruhe und einer Melancholie zu spüren, welche 1914 zum vollen Ausdruck kommen. Der Krieg markiert nämlich einen zweiten Bruch und führt Beckmann zur Abwendung vom apollinischen Schönen: in dem Schock und in der Gewalt des Wirklichen sucht er nach der «schrecklichen» dionysischen Schönheit und nach der vitalen Intensität, wodurch es ihm gelingt, die Katharsis der Kunst gewordenen schrecklichen Existenz vorzunehmen. Die dritte Phase markiert schließlich ab den zwanziger Jahren die metaphysische Überwindung des Dualismus des Schönen und Hässlichen: Beckmann entwickelt seine «Metaphysik der Objektivität», wodurch er die Welt transzendiert, das im Sichtbaren steckende Unsichtbare entdeckt und die mystische Einheit der durch die Kunst mit dem Subjet versöhnten Welt wiederherstellt. Dieses wandlungsfähige Wesen verkörpert also den Übergang von einer noch akademischen Vorstellung des Schönen zur pathetischen Kunst, die das Hässliche einschließt, bis zur metaphysischen Kunst, die den Widerspruch des Schönen und Hässlichen transzendiert, indem sie diese in eine Ästhetik des modernen Erhabenen integriert.

\section{INDEX}

Mots-clés : peinture, beauté, laideur

\section{AUTEURS}

\section{FLORENCE BANCAUD}

Université de Rouen 